\title{
Processo de alta hospitalar de pessoas com acidente vascular cerebral: uma revisão narrativa de literatura
}

\author{
Hospital discharge process for people with brain vascular accident: a narrative literature review \\ Proceso de alta hospitalaria para personas con accidente vascular cerebral: revisión narrativa de la
}

\section{literatura}

Recebido: 21/12/2021 | Revisado: 28/12/2021 | Aceito: 05/01/2022 | Publicado: 05/01/2022

Fernanda Seeber

ORCID https://orcid.org/0000-0002-6762-9984 Universidade do Planalto Catarinense, Brasil E-mail: seeberfernanda@gmail.com

Camille Peixer

ORCID: https://orcid.org/0000-0001-8509-2896 Universidade do Planalto Catarinense, Brasil E-mail: camillepeixer@uniplaclages.edu.br

Lilia Aparecida Kanan

ORCID: https://orcid.org/0000-0001-6412-0544 Universidade do Planalto Catarinense, Brasil E-mail: prof.lak@uniplaclages.edu.br

Anelise Viapiana Masiero

ORCID: https://orcid.org/0000-0003-3203-5876 Universidade do Planalto Catarinense, Brasil E-mail: anemasiero@uniplaclages.edu.br

\begin{abstract}
Resumo
O estudo objetivou a realização de uma revisão narrativa de literatura sobre o processo de alta hospitalar de pessoas com Acidente Vascular Cerebral (AVC). A busca foi realizada nas bases de dados, PubMed e Google Acadêmico, entre os meses de julho e agosto de 2021. Foram utilizados os descritores: "Stroke", "discharge planning" OR "Transitional Care" AND "Caregivers". Como critério de inclusão considerou-se publicações que abordaram o tema, publicadas entre os anos de 2016 a 2021, escritos em idioma português ou inglês e com acesso online. A pré-seleção considerou a leitura de título e resumo das publicaçãoes e classificou ao final 11 para leitura completa. Como principais resultados encontrou-se que o profissional mais envolvido no processo de alta hospitalar de pacientes acometidos por AVC é o enfermeiro (a). Entretanto, parece haver dificuldades no processo de transição do cuidado, principalmente no que se refere a comunicação e envolvimento dos pacientes e cuidadores na tomada de decisão. O planejamento da alta representa estratégia essencial para a transferência do paciente do hospital para casa e pode contribuir para a diminuição: das taxas de reinternação, das complicações, do tempo de internação hospitalar, dos custos de atendimento e de mortalidade. Ainda, destaca-se importância da educação em saúde e do trabalho interprofissional para o cuidado integral de pessoas com AVC.
\end{abstract}

Palavras-chave: Acidente Vascular Cerebral; Planejamento de alta; Cuidado transicional; Cuidadores.

\begin{abstract}
The study aimed to carry out a narrative literature review on the hospital discharge process of people with stroke. The search was performed in the databases, PubMed and Academic Google, between July and August 2021. The descriptors used were: "Stroke", "discharge planning" OR "Transitional Care" AND "Caregivers". As an inclusion criterion, publications that addressed the topic, published between the years 2016 to 2021, written in Portuguese or English and with online access, were considered. The pre-selection considered the reading of the title and abstract of the publications and classified at the end 11 for full reading. As main results, it was found that the professional most involved in the hospital discharge process of patients affected by stroke is the nurse. However, there seem to be difficulties in the care transition process, especially with regard to communication and involvement of patients and caregivers in decision-making. Discharge planning represents an essential strategy for transferring the patient from the hospital to the home and can contribute to the reduction of: readmission rates, complications, length of hospital stay, care costs and mortality. Still, the importance of health education and interprofessional work for the comprehensive care of people with stroke is highlighted.
\end{abstract}

Keywords: Stroke; Discharge planning; Transitional Care; Caregivers. 


\section{Resumen}

El estudio tuvo como objetivo realizar una revisión narrativa de la literatura sobre el proceso de alta hospitalaria de personas con accidente cerebrovascular (ACV). La búsqueda se realizó en las bases de datos PubMed y Academic Google, entre los meses de julio y agosto de 2021. Se utilizaron los descriptores: "Ictus", "Planificación del alta" O "Transitional Care" Y "Cuidadores". Como criterio de inclusión, se consideraron las publicaciones que abordaron el tema, editadas entre los años 2016 a 2021, escritas en portugués o inglés y con acceso online. La preselección consideró la lectura del título y resumen de los artículos y clasificó al final 11 publicaciones para lectura completa. Como principales resultados se encontró que el profesional más involucrado en el proceso de alta hospitalaria de los pacientes afectados por ictus es el enfermero (a). Sin embargo, parece haber dificultades en el proceso de transición asistencial, especialmente en lo que respecta a la comunicación y la implicación de pacientes y cuidadores en la toma de decisiones. La planificación del alta representa una estrategia fundamental para el traslado del paciente del hospital al domicilio y puede contribuir a la reducción de: tasas de reingreso, complicaciones, duración de la estancia hospitalaria, costes asistenciales y mortalidad. Aun así, se destaca la importancia de la educación sanitaria y el trabajo interprofesional para la atención integral de las personas con ictus.

Palabras clave: Accidente Cerebrovascular; Alta planificación; Cuidado de transición; Cuidadores.

\section{Introdução}

O Brasil encontra-se em uma transição demográfica significativa em razão do aumento da longevidade, da eficácia dos serviços de saúde, da baixa fecundidade e da melhoria da qualidade de vida em geral (Vasconcelos \& Gomes, 2012).

Sob outra perspectiva, a adoção de um estilo de vida não-adequado (tabagismo, má alimentação, inatividade física, consumo de álcool e outras drogas) associado a fatores não modificáveis (sexo, idade e herança genética) elevam o risco de Doenças Crônicas Não Transmissíveis (DCNT) (Malta et al., 2015). As DCNT constituem sete das dez principais causas de morte no mundo, conforme estimativas globais de saúde apontadas pela Organização Mundial da Saúde - OMS (2020). Dentre as DCNT, o Acidente Vascular Cerebral (AVC) representa a principal causa de incapacidade não somente no mundo, mas também no Brasil (OPAS, 2020), com uma incidência anual de 108 casos para cada 100 mil habitantes (OMS, 2013).

Segundo Safanelli et al. (2019), o custo médio para o tratamento de um paciente com qualquer tipo de AVC em hospital público gira em torno a 10 mil reais. Além das complicações e sequelas, o paciente hospitalizado por AVC, tem o dobro de chances de óbito, quando comparado a pacientes internados com outras enfermidades (Datasus, 2018), o que destaca essa patologia como um importante problema de saúde pública.

As pessoas acometidas por AVC, em geral, apresentam alguma incapacidade funcional como sequelas dependendo da área afetada, do grau de lesão e da capacidade de recuperação do indivíduo que podem ser temporárias ou permanentes (Rodrigues et al., 2013).

O nível de incapacidade do paciente determina o grau de dependência (cognitiva e motora), o que leva a um declínio na qualidade de vida e afeta o cotidiano do paciente e de seus familiares (Mayor, 2009; Rodrigues et al., 2013). Alterações discretas são manifestadas por $37 \%$ dos pacientes após o acidente vascular cerebral, $16 \%$ apresentam moderada incapacidade e 32\% demonstram alteração intensa ou grave da capacidade funcional. Alguns, inclusive, passam a depender de cadeira de rodas ou ainda, ficam restritos ao leito. Somente $15 \%$ dos pacientes não apresentam prejuízo da capacidade funcional (Giles \& Rothwell, 2008).

Para identificação do nível de acometimento das funções a avaliação da pessoa que sofreu um ou mais eventos de AVC deve ser feita por equipe multidisciplinar. É necessário que os vários aspectos potencilamente comprometidos sejam abordados da forma mais sinérgica e completa possível (Mello, 2013).

Durante a internação hospitalar, a equipe multidisciplinar é a responsável pelo cuidado. Entretanto, no retorno para casa é a família quem assume essa responsabilidade, algo que marca a transição do curar para o cuidar em casa. É nesse momento que a família ao enfrentar as mudanças, tanto na capacidade funcional, quanto na imagem corporal do sobrevivente após AVC, deve auxiliar na recuperação da doença e no apoio para o autocuidado (Rodrigues et al., 2013). 
Em virtude da necessidade de diminuir o tempo de internação, devido aos custos e riscos associados, a alta hospitalar precoce tem sido adotada como estratégia. A alta precoce visa beneficiar o paciente e exige que as orientações a ele e/ou seus cuidadores sejam baseadas, em uma avaliação de suas necessidades. Para tal, devem ser considerados o contexto socioeconômico, as restrições físicas e psíquicas e o ambiente em que vive (Brasil, 2012).

Neste contexto, a OMS destaca a importância do planejamento de alta hospitalar ao paciente como diretriz básica para transferência adequada de cuidados (Brasil, 2013). Portanto, quanto mais cedo começarem as orientações e o plano de alta, menor será a incidência de reinternação hospitalar, assim como o tempo de permanência e readmissões. A alta planejada pode não apenas melhorar significativamente a qualidade de vida do paciente, mas também alcançar com êxito a transição do cuidado (Fox et al., 2013).

Diante do exposto, o objetivo do presente estudo esteve centrado na realização de uma revisão narrativa de literatura sobre o processo de alta hospitalar de pessoas com acidente vascular cerebral.

\section{Metodologia}

O presente estudo se caracteriza como um estudo de revisão narrativa de literatura, a qual não tem obrigatoriedade de apresentar uma questão específica e permite uma busca mais ampliada, na qual a seleção dos artigos está sujeita a subjetividade dos pesquisadores sem esgotar exaustivamente as fontes de informação (Cordeiro et al.2007). Mesmo não sendo uma exigência do método, neste estudo optou-se por pré-determinar as bases de busca assim como, foram estabelecidos critérios de inclusão para a pré-seleção das Publicações.

A busca foi realizada nas bases de dados do PubMed e Google Acadêmico, entre os meses de julho e agosto de 2021. Foram utilizados os descritores: "Stroke", "Discharge Planning" OR "Transitional Care" AND "Caregivers". Foram analisadas publicações que abordassem o tema, publicadas entre os anos de 2016-2021) escritos em idioma português ou inglês e com acesso online e livre.

Inicialmente as publicações foram selecionadas pelo. Posteriormente as pré-selecionadas foram avaliadas na íntegra por todos os autores do estudo. A figura 1 apresenta o fluxograma do processo de seleção. A partir da seleção final foram utilizadas 11 publicações para leitura completa e criteriosa e coleta de dados que foram inseridas em um quadro considerando os seguintes aspectos: objetivo, tipo de estudo, principais resultados e conclusões. Os resultados são apresentados e discutidos de maneira descritiva.

Figura 1 - Síntese do processo de seleção dos estudos para análise.

Estudos potencialmente elegíveis identificados pela busca nas bases de dados $=10.640$

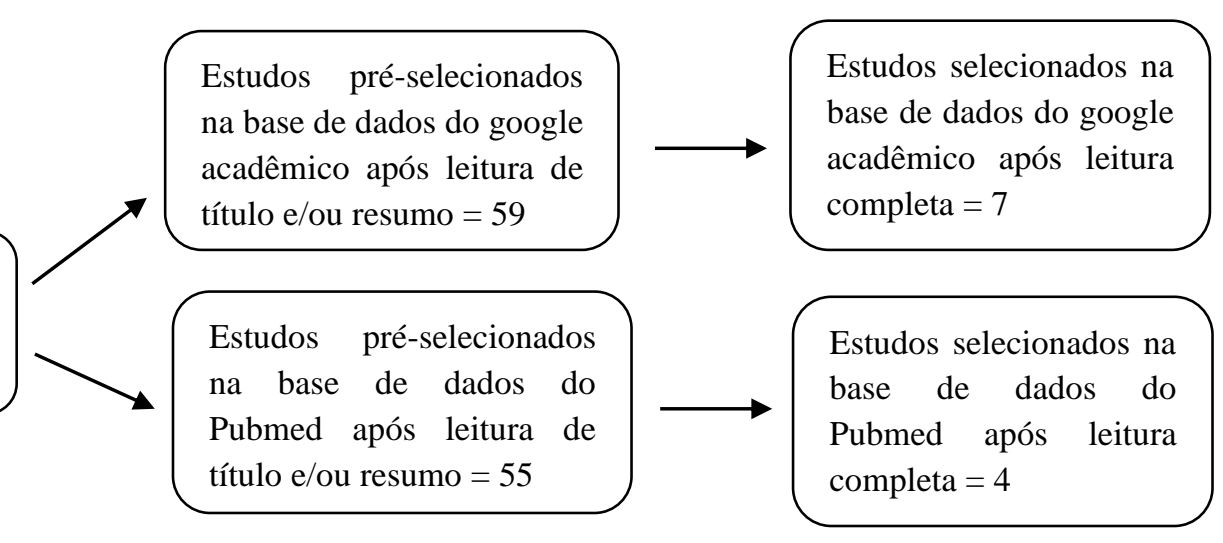

Fonte: Autores. 


\section{Resultados}

Dentre os 11 estudos selecionados estão estudos de revisão integrativa, sistemática, estudos quantitativos, qualitativos, relatos de caso e estudo clínico randomizado. O Quadro 1, apresenta as principais informações extraídas dos estudos no que se refere aos objetivos, metodologias, principais resultados e conclusões. No que se refere aos objetivos dos estudos destacam-se aqueles que buscaram investigar as perspectivas dos pacientes, cuidadores e profissionais de saúde (principalmente da área da enfermagem) sobre o processo ou planejamento de alta hospitalar; outros estudos buscaram compreender os fatores que influenciam o planejamento de alta e por fim, estudos que relatam ou comparam modelos de assistência durante esta transição do cuidado do ambiente hospitalar para o ambiente familiar.

Na análise da percepção dos pacientes, cuidadores e profissionais de saúde sobre o processo de planejamento observa-se que os primeiros (pacientes e cuidadores) relatam dificuldade de comunicação e pouco envolvimento na tomada de decisão sobre os cuidados. Já os profissionais enfatizam a importância do conhecimento para elaboração adequada do planejamento e que este era consequência de treinamento especializado. Alguns enfermeiros atribuem aos familiares as dificuldades no repasse das informações por falta de conhecimento da doença. Ainda, destacam que os profissionais deveriam ter iniciativas para evitar intervenções desnecessárias e que incluir todos os cuidados que o paciente de AVC necessita representa um desafio.

Quando analisado o profissional mais envolvido no processo de alta as citações convergem para o enfermeiro e, dependendo do modelo de assistência, muitas vezes o próprio paciente ou familiares/cuidadores influenciam mais no planejamento de alta do que os médicos. Verifica-se também, que em países como os Estados Unidos, por exemplo, vigora um modelo de assistência à saúde diferente do modelo brasileiro: o seguro de saúde e a qualidade do serviço de cuidado pós fase aguda foram os principais fatores de seleção do local para continuidade do tratamento, sendo o seguro a principal barreira. Ainda, encontra-se que a pressão para a alta precoce impacta a decisão final dos pacientes, o que faz com que fatores não clínicos sejam os principais influenciadores na decisão do cuidado pós fase aguda. O planejamento da alta contribui para diminuir as taxas de reinternação, complicações, tempo de internação hospitalar e custos de atendimento e mortalidade. Além disso, essas intervenções podem aumentar a qualidade de cuidado, conhecimento do paciente do cuidador e dos profissionais de saúde. 
Research, Society and Development, v. 11, n. 1, e36911125016, 2022

(CC BY 4.0) | ISSN 2525-3409 | DOI: http://dx.doi.org/10.33448/rsd-v11i1.25016

\begin{tabular}{|c|c|c|c|c|}
\hline & Autores (Ano) & Objetivo principal & Tipo de estudo & Principais Resultados \\
\hline 1. & Brady et al.(2016) & $\begin{array}{l}\text { Investigar as perspectivas dos pacientes e } \\
\text { também da equipe de enfermagem sobre a } \\
\text { implementação de uma intervenção de } \\
\text { cuidado para pacientes com incontinência } \\
\text { urinária após o AVC. }\end{array}$ & $\begin{array}{l}\text { Estudo } \\
\text { qualitativo }\end{array}$ & $\begin{array}{l}\text { Percepção dos pacientes: (a) desafios de comunicação; (b) percepções mistas de } \\
\text { cuidados; (c) ambiguidade de enfoque entre questões de mobilidade e de continência; e } \\
\text { (d) inconsistente envolvimento na tomada de decisões sobre cuidados. }\end{array}$ \\
\hline 2. & $\begin{array}{l}\text { Gibson et al. } \\
\text { (2016) }\end{array}$ & $\begin{array}{l}\text { Explorar as opiniões dos sobreviventes do } \\
\text { AVC, cuidadores e enfermeiros sobre as } \\
\text { experiências de a adesão à medicação após } \\
\text { a alta hospitalar. }\end{array}$ & $\begin{array}{l}\text { Estudo } \\
\text { qualitativo }\end{array}$ & $\begin{array}{l}\text { Os fatores que afetam a adesão incluíram depressão, benefícios imperceptíveis e } \\
\text { preocupações com efeitos adversos das medicações. Os enfermeiros sugeriram que a } \\
\text { baixa adesão poderia ser mais provável em pacientes que vivem sozinhos ou com } \\
\text { histórico prévio de não adesão. Outro fator seria a dificuldade de tomar a medicação } \\
\text { por limitações impostas pelo AVC: estes incluíam deficiências, cognição, polifarmácia } \\
\text { e falta de informação. }\end{array}$ \\
\hline 3. & $\begin{array}{l}\text { Magdon-Ismail et } \\
\text { al. (2016) }\end{array}$ & $\begin{array}{l}\text { Compreender os fatores que influenciam o } \\
\text { planejamento de alta de pacientes com } \\
\text { AVC atendidos em hospitais do Nordeste } \\
\text { dos Estados Unidos }\end{array}$ & $\begin{array}{l}\text { Estudo } \\
\text { quantitativo }\end{array}$ & $\begin{array}{l}\text { Participaram enfermeiros e assistentes sociais com média de } 20 \text { anos de experiência. } \\
\text { Destes, } 69 \% \text { dos hospitais possuíam uma unidade de AVC designada para o cuidado } \\
\text { do paciente com AVC. } \\
\text { Os pacientes e familiares influenciavam mais que os médicos ou outros profissionais } \\
\text { no planejamento de alta. Os outros profissionais da equipe eram mais influentes que } \\
\text { os médicos. O seguro de saúde e a qualidade do serviço de cuidado pós fase aguda } \\
\text { foram os principais fatores de seleção do local, sendo o seguro a principal barreira. } \\
\text { Mais de } 50 \% \text { dos participantes reportaram que a pressão para a alta precoce impacta na } \\
\text { decisão final dos pacientes. Fatores não clínicos foram percebidos como principais } \\
\text { influenciadores na decisão do cuidado pós fase aguda. }\end{array}$ \\
\hline 4. & $\begin{array}{lll}\text { Gesell } & \text { et } & \text { al. } \\
(2017) & & \end{array}$ & $\begin{array}{l}\text { Comparar a eficácia comparativa de um } \\
\text { modelo de cuidado transitório centrado no } \\
\text { paciente que fornece estrutura e processos } \\
\text { para o cuidado pós-agudo versus modelo } \\
\text { de alta habitual. }\end{array}$ & $\begin{array}{l}\text { Estudo clinico } \\
\text { randomizado }\end{array}$ & $\begin{array}{l}\text { O estudo Comprehensive Post-Acute Stroke Services (COMPASS) é um estudo que } \\
\text { propõe um modelo de intervenção precoce de suporte após a alta hospitalar do paciente } \\
\text { com AVC, já implementado como prática no Canada e Europa. Essa intervenção } \\
\text { inovadora combina serviços do cuidado Pós-AVC envolvendo os serviços de saúde da } \\
\text { comunidade para aprimorar a continuidade do cuidado. Os pacientes são contactados } \\
\text { por telefone } 2,7,14,30 \text { e } 60 \text { dias após a alta, além das visitas clínicas em } 7 \text { e } 14 \text { dias. }\end{array}$ \\
\hline 5. & $\begin{array}{l}\text { Santos et al. } \\
(2021)\end{array}$ & $\begin{array}{l}\text { Analisar a atuação do enfermeiro na } \\
\text { assistência ao paciente com Acidente } \\
\text { Vascular Cerebral na alta hospitalar e } \\
\text { investigar as práticas realizadas pelos } \\
\text { enfermeiros no repasse das informações } \\
\text { aos cuidadores sobre o cuidado domiciliar } \\
\text { de paciente com AVC na alta hospitalar. }\end{array}$ & $\begin{array}{l}\text { Revisão de } \\
\text { Literatura }\end{array}$ & $\begin{array}{l}\text { Existe uma dicotomia em relação às dificuldades no ensinamento sobre a assistência ao } \\
\text { paciente acometido por AVC repassada aos cuidadores/familiares. Alguns enfermeiros } \\
\text { atribuem aos familiares as dificuldades no repasse das informações por falta de } \\
\text { conhecimento da doença. } \\
\text { Outros relatam que a maior dificuldade está relacionada às várias atribuições } \\
\text { dificultando o repasse das informações, bem como, a falta de um protocolo de ação } \\
\text { para assistência de enfermagem, para realizar os cuidados de maior complexidade } \\
\text { técnica. }\end{array}$ \\
\hline 6. & $\begin{array}{l}\begin{array}{l}\text { Buzarelo et } \\
(2018)\end{array} \\
\text { al. }\end{array}$ & $\begin{array}{l}\text { Aplicar o Modelo Calgary de Avaliação } \\
\text { Familiar no contexto domiciliar em família } \\
\text { que convive com pessoa acometida por } \\
\text { Acidente Vascular Encefálico. }\end{array}$ & $\begin{array}{l}\text { Estudo de caso, } \\
\text { qualitativo }\end{array}$ & $\begin{array}{l}\text { A construção do genograma e do ecomapa, permitiu à compreensão das relações } \\
\text { familiare, da interação entre os membros e as redes sociais de apoio à família. Este } \\
\text { grupo peculiar lidava diariamente com questões complexas que extrapolavam as } \\
\text { limitaçôes oriundas do AVE, como a dependência de drogas (lícitas e ilícitas), os } \\
\text { transtornos mentais, o isolamento, os conflitos. }\end{array}$ \\
\hline
\end{tabular}

\section{Principais Conclusões}

Pacientes (principalmente os com incontinência urinária grave) descreveram desafios da comunicação envolvimento nas decisões sobre cuidados. Em contraste, as enfermeiras descreveram melhoria do conhecimento, atitudes e confiança de continência, juntamente com uma mudança para abordagens reabilitativas.

Sobreviventes de AVC e cuidadores informais não têm conhecimento e apoio necessários para gerenciar a medicação após a alta. As oportunidades das ex a pexpos de adesão de medicamentos para apoiar sobreviventes de AVC $\log 0$ após a alta.

O estudo sugere que fatores não clínicos muitas vezes desempenham um papel desproporcional na seleção do cuidado pós-tratamento para pacientes com AVC talvez seja necessária uma revisão do sistema de atenção ao AVC.

Este modelo de assistência ao paciente com AVC avaliar seu estado funcional e necessidades de cuidados pós fase aguda e fornece a base para um plano de atendimento eletrônico individualizado atendendo a necessidades específicas.

Há necessidade da presença do cuidador/familiar com suporte necessário para evitar reinternações.

enfermeiro tem uma participac̃o significativa na orientação do cuidador.

A orientação deve ser feita de forma clara e linguagem acessível.

$\mathrm{O}$ modelo proposto permitiu avaliar a família em sua multidimensionalidade, e auxiliá-la e fortalecê-la diante das adversidades.

$\mathrm{O}$ processo de adoecimento modifica, consolida ou fragiliza os vínculos, resultando em sofrimento 
Research, Society and Development, v. 11, n. 1, e36911125016, 2022

\begin{tabular}{|c|c|c|c|c|c|}
\hline & & & & $\begin{array}{l}\text { Proporcionando que a família reorganize as funções primárias, renegociação de } \\
\text { compromissos emocionais, financeiros e de atividades domésticas. Espiritualidade } \\
\text { aparece como um fator importante para o enfrentamento das situações. }\end{array}$ & $\begin{array}{l}\text { psicossocial para o grupo de uma maneira geral. } \\
\text { A família pode ser provedora de cuidados, mas } \\
\text { necessita de assistência. } \\
\text { Desenvolver estratégias e acompanhamento das familias } \\
\text { no período imediato a alta hospitalar, pois este } \\
\text { configura-se como fase de reorganização do grupo } \\
\text { familiar. }\end{array}$ \\
\hline 7. & $\begin{array}{l}\text { Da Silva Azevedo } \\
\text { et al. (2019) }\end{array}$ & $\begin{array}{l}\text { Caracterizar } \quad \text { o nível funcional } \text { e } \\
\text { dependências na alta dos cuidados } \\
\text { intensivos e verificar o momento de início } \\
\text { das intervenções de enfermagem na } \\
\text { reabilitação dos pacientes. }\end{array}$ & $\begin{array}{l}\text { Estudo } \\
\text { descritivo/ } \\
\text { exploratório, } \\
\text { quantitativo }\end{array}$ & $\begin{array}{l}\text { São prestados cuidados de reabilitação funcional motora, inicialmente mobilização } \\
\text { passiva, progredindo para resistida, até ao levante. Exercícios de marcha só são } \\
\text { realizados em doentes extubados. Simultaneamente, são prestados cuidados de } \\
\text { reabilitação funcional respiratória, com a finalidade de facilitar o "desmame } \\
\text { ventilatório". }\end{array}$ & $\begin{array}{l}\text { No momento da alta verificaram-se elevados níveis de } \\
\text { dependência funcional no autocuidado básico e } \\
\text { mobilidade/transferências, sendo necessário melhorar a } \\
\text { sua caraterização para a continuidade de cuidados de } \\
\text { reabilitação. A mobilização dos doentes críticos deve } \\
\text { acontecer precocemente. }\end{array}$ \\
\hline 8. & Chen et al. (2020) & $\begin{array}{l}\text { Identificar desafios e oportunidades para } \\
\text { sobreviventes de AVC e cuidadores no } \\
\text { hospital para cuidados de transição } \\
\text { domiciliar. }\end{array}$ & $\begin{array}{l}\text { Revisão } \\
\text { sistemática }\end{array}$ & $\begin{array}{l}\text { Os sistemas de saúde e assistência social, influenciam os cuidados, permitindo que } \\
\text { sobreviventes e cuidadores de AVC gerenciem os cuidados de transição. } \\
\text { Em segundo lugar, a parceria dos profissionais de saúde com sobreviventes e } \\
\text { cuidadores de AVC decide em grande parte o apoio personalizado a eles. } \\
\text { Em terceiro lugar, sobreviventes e cuidadores estão em diferentes níveis aceitação para } \\
\text { lidar com desafios. }\end{array}$ & $\begin{array}{l}\text { Sobreviventes e cuidadores de AVC encontram enormes } \\
\text { desafios na autogestão do hospital para o cuidado de } \\
\text { transição domiciliar. }\end{array}$ \\
\hline 9. & $\begin{array}{lll}\begin{array}{l}\text { Loureiro } \\
(2020)\end{array} & \text { et } & \text { al. }\end{array}$ & $\begin{array}{l}\text { Compreender o processo de planejamento } \\
\text { de alta na perspectiva dos enfermeiros e, } \\
\text { analisar as diferenças de planejamento de } \\
\text { cuidados, de acordo com a formação } \\
\text { acadêmica e especialidade. }\end{array}$ & $\begin{array}{l}\text { Estudo } \\
\text { qualitativo }\end{array}$ & $\begin{array}{l}\text { Da análise emergiram as seguintes áreas temáticas: o processo de planejamento de alta, } \\
\text { a continuidade de cuidados, os instrumentos de apoio ao planejamento de alta, as boas } \\
\text { práticas e o modelo em uso. } \\
\text { Os enfermeiros desenvolvem o planejamento de alta de forma pouco organizada e } \\
\text { sistematizada, desprovida de instrumentos de medição, e sem fundamentação teórica } \\
\text { na sua tomada de decisão. } \\
\text { Não há uma articulação sustentada ao longo do processo de planeamento de alta, entre } \\
\text { o momento em que é iniciado e a alta efetiva do doente. }\end{array}$ & $\begin{array}{l}\text { Os enfermeiros que manifestaram maior envolvimento } \\
\text { no processo e utilização de recursos, como os } \\
\text { instrumentos de medida, embora restritos ao seu âmbito } \\
\text { profissional, foram os enfermeiros especialistas de } \\
\text { reabilitação. }\end{array}$ \\
\hline 10. & $\begin{array}{l}\text { Suksatan \& Posai } \\
(2020)\end{array}$ & $\begin{array}{l}\text { Examinar os efeitos das } \\
\text { Intervenções de planejamento de alta } \\
\text { hospitalar em pacientes tailandeses com } \\
\text { AVC. }\end{array}$ & $\begin{array}{l}\text { Revisão } \\
\text { integrativa }\end{array}$ & $\begin{array}{l}\text { Os resultados indicaram que os planejamentos de alta hospitalar para a qualidade do } \\
\text { atendimento em pacientes tailandeses com AVC foram desenvolvidos e conduzidos } \\
\text { apenas recentemente, e a maioria ainda está em teste com alguns pacientes. } \\
\text { O planejamento de alta hospitalar pode diminuir as taxas de reinternação, } \\
\text { complicações, tempo de internação hospitalar e custos de atendimento e mortalidade. } \\
\text { Além disso, essas intervenções podem aumentar a qualidade de cuidado, conhecimento } \\
\text { do paciente do cuidador e dos profissionais de saúde. }\end{array}$ & $\begin{array}{l}\text { Os planejamentos fornecem informações básicas para } \\
\text { alta para pacientes com AVC, preparando cuidadores e } \\
\text { familiares usando educação para a alta hospitalar } \\
\text { coordenada por uma equipe multidisciplinar de cuidado. } \\
\text { O planejamento da alta é uma estratégia essencial para a } \\
\text { transferência do paciente do hospital para casa. }\end{array}$ \\
\hline 11. & Silva et al. (2021) & $\begin{array}{l}\text { Realizar a Assistência a um paciente com } \\
\text { sequelas de Acidente Vascular Cerebral } \\
\text { acompanhado pelo Programa Melhor em } \\
\text { Casa. }\end{array}$ & Estudo de caso & $\begin{array}{l}\text { O Programa Melhor em Casa é um serviço indicado para pessoas que apresentam } \\
\text { dificuldades temporárias ou definitivas de sair do espaço de sua casa para chegar até } \\
\text { uma unidade de saúde, ou ainda para pessoas que estejam em situações nas quais a } \\
\text { atenção domiciliar é a mais indicada para o seu tratamento, como em casos de } \\
\text { pacientes com sequelas de Acidente Vascular Cerebral. } \\
\text { O atendimento é feito pela Equipe Multidisciplinar de Atenção Domiciliar, formada } \\
\text { prioritariamente por médicos, enfermeiros, técnicos em enfermagem, fisioterapeuta } \\
\text { e assistente social. Outros profissionais, como: fonoaudiólogo, nutricionista, } \\
\text { odontólogo, psicólogo, terapeuta ocupacional e farmacêutico,poderão compor a } \\
\text { Equipe Multidisciplinar de Apoio. }\end{array}$ & $\begin{array}{l}\text { A esta estratégia é eficaz quando empregada aos } \\
\text { cuidados de enfermagem dos serviços de atenção } \\
\text { domiciliar, proporcionando uma assistência holística e } \\
\text { com embasamento científico. }\end{array}$ \\
\hline
\end{tabular}

Fonte: Elaborado pelos autores (2021). 


\section{Discussão}

No Brasil, entre os anos de 2018 e 2021 foram registradas 495.558 internações por AVC isquêmico ou hemorrágico, segundo dados do Sistema de Informações Hospitalares do SUS (SIH/SUS) do Ministério da Saúde (Brasil, 2021). Além de todos os fatores de risco anteriormente descritos para a ocorrência do AVC, a partir do final de 2019, mais um agravante se soma as estatísticas não satisfatórias dos AVCs. A pandemia pelo novo Coronavírus humano, chamada de Síndrome Respiratória Aguda Grave do Coronavírus 2 (SARS-CoV-2) ou (COVID-19) com mais de 266 milhões de casos no mundo e mais de 5 milhões de mortos, tem desafiado a humanidade e as autoridades de saúde (WHO, 2021).

Um estudo recente sobre as características e prognóstico de pacientes com COVID e quadros agudos de AVC isquêmico, realizado a partir de 28 sistemas de registro de 16 países, sugere uma associação entre o COVID-19 e os AVCs isquêmicos, e que estes são mais severos com piores perdas funcionais e maior mortalidade (Ntaios, 2020).

Pressupõe-se que o mecanismo de ocorrência do AVC, causado pelo COVID-19, pode ser explicado através da interação entre os fatores de risco convencionais de AVC, infecção, resposta inflamatória sistêmica e desestabilização da placa. Marcadores inflamatórios, elevação do dímero D e aumento da atividade das citocinas foram observados em pacientes com COVID-19 (Bhatia \& Srivastava, 2020).

Na presente revisão observou-se, em nível internacional, modelos de assistência e de transição do cuidado já instituídos em países europeus e da América do Norte (Gesell et al., 2017). No Brasil, um marco importante é instituição da Rede de Urgência e Emergência (RUE), sendo umas das prioridades a implantação das linhas de cuidado do AVC por meio da Portaria n. ${ }^{\circ} 665$, de 12 de abril de 2012 (Brasil, 2012).

A linha de cuidado, traz padronizações técnicas relativas à organização do atendimento no sistema de saúde. Descreve o caminho mais adequado que o paciente deve percorrer ou o encaminhamento pela rede de assistência que a equipe de saúde deve prescrever. O conteúdo tem informações relativas às ações e atividades de promoção, prevenção, tratamento e reabilitação a serem desenvolvidas por equipe multidisciplinar em cada serviço de saúde (Brasil, 2019).

Assim, ao promover a integração de ações e serviços, caracterizada pela formação de relações horizontais desde a unidade de saúde na Atenção Primária até os serviços especializados, se fortalece a Rede de Atenção à Saúde (RAS) (Oliveira, 2016). Ainda, dentro da linha de cuidado ao AVC está prevista a atenção especializada de maior complexidade em Unidades especializadas de Cuidado Agudo ao AVC (U-AVCs) (Luiza et al., 2013).

As U-AVCs estão classificadas em três níveis, tipo I, II e III. As unidades tipo III são as que praticam o cuidado integral, multiprofissional com, no mínimo, 10 leitos, coordenada por neurologista, dedicada ao cuidado dos pacientes acometidos pelo AVC (isquêmico ou hemorrágico) até quinze dias da internação hospitalar. Esta unidade tem a atribuição de dar continuidade ao tratamento da fase aguda, reabilitação precoce e investigação etiológica completa, o que prepara o paciente e família para o retorno domiciliar (Brasil, 2012).

A equipe multiprofissional que compõe as U-AVCs realiza frequentemente, por meio de reuniões, a avaliação, discussão e direcionamento do quadro clinico e terapêutico do paciente, sendo estes aspectos importantes para auxiliar no entendimento do sujeito e do cenário abrangido pelo AVC. Essas ações são importantes para subsidiar a elaboração do Plano Terapêutico Singular (PTS), visando dessa maneira uma perspectiva global que atenda às necessidades de cada pessoa (Brasil, 2013).

Os protocolos baseados no PTS de cada paciente irão nortear o atendimento além de dar suporte científico para a equipe. Ainda, proporcionam a evolução no cuidado com o paciente, o que promove melhor qualidade e eficiência no atendimento e consolida o profissional como um elo assertivo e decisório no processo de cuidar (Pinto et al., 2011). Na rotina diária das U-AVCs, as orientações de alta hospitalar para uma desospitalização efetiva, iniciam desde o primeiro contato com 
paciente e familiares, e se efetivam durante todos o período de hospitalização. Este processo é organizado pela equipe com conhecimento amplo do quadro clinico e do possível prognóstico do paciente (Brasil, 2013).

Neste planejamento é elaborado um resumo conciso e instrutivo sobre as informações necessárias ao cuidado em domicilio da pessoa com AVC. Estas informações são entregues ao paciente e familiares no intuito de contribuir com o cuidado e evitar novas reinternações (Pinheiro, 2018). Porém, mesmo com todo o empenho da equipe, podem surgir obstáculos que impossibilitem a aplicação dos protocolos de orientação de alta hospitalar. Citam-se como exemplos a disponibilidade de tempo, a grande demanda de pacientes, a rotatividade de pacientes, ou ainda, os horários da alta hospitalar (Gracioto, 2006).

Os estudos utilizados nessa revisão também apontam dificuldades no processo de transição do cuidado, por exemplo na adesão dos medicamentos de uso necessário após a alta hospitalar (Gibson et al. 2016). Segundo estes autores, fatores como depressão, benefícios imperceptíveis, preocupações com efeitos adversos das medicações, viver sozinho, com histórico prévio de não adesão e as limitações impostas pelo próprio AVC poderiam estar entre as justificativas.

Ainda, na perspectiva de pacientes e cuidadores há dificuldade de comunicação e pouco envolvimento dos pacientes e cuidadores na tomada de decisão (Loureiro et al., 2020), que por vezes pode estar relacionados a dificuldade de compreensão dos familiares e cuidadores (Brady et al., 2016; Santos et al., 2021), possivelmente pelo desconhecimento da doença ou a dificuldade de incluir todos os cuidados necessários (Santos et al., 2021), visto que é comum que os pacientes recebam alta hospitalar com níveis significativos de dependência funcional no autocuidado básico (Da Silva Azevedo et al. 2019).

Outro ponto importante a destacar nesta fase de transição do cuidado é a reorganização das famílias pois envolve desde a reorganização das atividades básica à questões financeiras, emocionais e espirituais (Buzarelo et al. 2018). O processo de adoecimento modifica, consolida ou fragiliza os vínculos, resultando em sofrimento psicossocial para o grupo de uma maneira geral (Buzarelo et al. 2018).Sobreviventes e cuidadores estão em diferentes níveis aceitação para lidar com desafios, por isso a importância do apoio personalizado a eles por parte dos profissionais de saúde (Chen et al. 2020)

Segundo Brady et al. (2016) a elaboração adequada do planejamento está condicionada a um treinamento especializado Embora o planejamento possa ser feito por uma equipe multiprofissional, os enfermeiros são os profissionais mais envolvido no processo de alta. Esse dado pode ser compreendido a partir de Dusek et al .(2015), quando apontam o papel do enfermeiro (a) na transição do cuidado, pois este, atua na assistência direta ao paciente e possui conhecimentos clínicos e gerenciais necessários para realizar transições de forma adequada, ordenada, eficiente e eficaz.

Corroborando esse entendimento, Burke et al. (2013) destacam que a coordenação da transição do cuidado deve ser realizada pelo profissional que está em maior contato com o paciente/familiares na unidade hospitalar, pois ao acompanhar, avaliando seus aspectos globais, é facilitador da educação necessária para o autocuidado enquanto o paciente está internado. Além disso, são fatores que influenciam o planejamento de alta o modelo de assistência a saúde. Ainda, os autores reportam que a pressão para a alta precoce impacta na decisão final dos pacientes, o que faz com que fatores não clínicos sejam os principais influenciadores na decisão do cuidado pós fase aguda (Magdon-Ismail et al. 2016).

Fica evidente nos estudos que integraram a presente revisão narrativa, que o planejamento da alta é uma estratégia essencial para a transferência do paciente do hospital para casa e contribui para diminuir as taxas de reinternação, complicações, tempo de internação hospitalar e custos de atendimento e mortalidade (Suksatan \& Posai, 2020).Além disso, essas intervenções podem aumentar a qualidade de cuidado, conhecimento do paciente do cuidador e dos profissionais de saúde (Gesell et al. 2017; Gibson et al. 2016, Silva et al. 2021, Suksatan \& Posai, 2020).

Neste ínterim é pertinente destacar o Laboratório de Inovação para o Enfrentamento do Acidente Vascular Cerebral (LAB-AVC Serra Catarinense), reconhecido com experiência inovadora em Educação em Saúde pelo Ministério da Saúde e Organização Panamericana de Saúde (Brasil, 2018). Dentre vários objetivos do Laboratório estava contribuir assistência integral 
aos pacientes e familiares pós-alta hospitalar com AVC. Dentre as ferramentas utilizadas pela equipe interprofissional destacase o SISAVC®, sistema informatizado desenvolvido no Laboratório de Inovação para dar apoio para a referência e contrarreferência do paciente com AVC pós-alta hospitalar (Faria, 2018). O sistema abriga informações sociodemográficos condições clínicas, plano terapêutico singular e dados levantados pela equipe da Estratégia da Saúde da Família (Faria, 2018).

Com base no aumento do número de casos de AVC causados por COVID-19 e a necessidade de evitar a exposição ao vírus causada por períodos longos de internamento e reinternações, o processo de orientações de alta hospitalar se tornou ainda mais desafiador para as equipes interprofissionais (Crispim et al., 2020).

Crispim et al. (2020) relatam ainda que, frente a essa nova realidade as famílias têm ficado cada vez menos em contato com os pacientes hospitalizados para evitar o risco de contaminação. As visitas e permanência de acompanhantes com os pacientes tem sido restrita, o que faz com que os profissionais de saúde criem novas maneiras de comunicação com as equipes, através da orientação por telemedicina ou por cartilhas.

Novas rotinas tiveram que ser organizadas para a assistência de pacientes nas UAVC e para pacientes com sintomas de AVC E COVID-19. Em 2020, o Departamento Científico de Doenças Cerebrovasculares da Academia Brasileira de Neurologia, SBDCV e da Sociedade Brasileira de Neurorradiologia (2020) e OPAS (2020) organizaram organogramas específicos com recomendações no manejo de pacientes com sintomas de AVC e COVID, incluindo contato telefônico mensagens de texto SMS e vídeo chamadas.

Como nova alternativa de comunicação, a LAB AVC UFRJ (2020) elaborou um e-book com orientações de exercícios domiciliares para pacientes, acometidos por AVC em isolamento protetor devido à pandemia causada por COVID-19, destinado a familiares, cuidadores, amigos, profissionais ou qualquer pessoa que se disponha a prestar cuidados em saúde a pacientes com limitações físicas decorrentes de um AVC. Este é um dos exemplos, dentre outros, que reforça a importância da educação em saúde para a alta hospitalar coordenar, que contribui na preparação de cuidadores e familiares para a rotina do ambiente familiar (Suksatan \& Posai, 2020).

Pensar em uma assistência integral aos pacientes com AVC é, sem dúvida, um desafio a ser superado pela gestão dos serviços de saúde e perpassa por processos de formação articulados aos diversos níveis de atenção à saúde no intuito de oferecer uma assistência integral e longitudinalizada (Faria et al. 2019). Nesse sentido um modelo de à saúde centrada na interdisciplinaridade e integralidade pode ser uma estratégia efetiva Kanan et al. (2018) e uma maneira de operacionalizar a relação horizontal entre os profissionais, construindo linhas de transversalidade (Faria et al. 2019).

\section{Considerações Finais}

A leitura dos estudos incluídos nessa revisão possibilita constatar os profissionais responsáveis pela alta hospitalar de um paciente pós AVC devem se valer de habilidades educacionais de modo a orientar familiares/cuidadores para um cuidado humanizado, voltado ao bem-estar e à recuperação do paciente. Em outros termos: é preciso que o processo de alta hospitalar seja realizado de modo a assegurar a compreensão dos familiares/cuidadores para que possa ser minimizado o sentimento de desamparo que naturalmente os acomete. Tal cuidado, é contributivo à recuperação dos pacientes acometidos por AVC, algo que tende a evitar novas hospitalizações.

As unidades especializadas para o tratamento agudo dos pacientes com acidente vascular cerebral (U-AVCs) têm por finalidade o cuidado integral interprofissional e por atribuição dar continuidade ao tratamento da fase aguda, por meio reabilitação precoce preparando o paciente e família para o retorno para casa com um plano terapêutico singular. Infelizmente o quantitativo destas unidades é insuficiente em relação a demanda do país. Neste contexto, seria interessante desenvolver estudos futuros que avaliassem se a implantação de UAVCs aprimorou o processo de alta hospitalar e contribui para a melhoria da 
assistência.

\section{Agradecimentos}

Agradecemos o apoio financeiro do Governo do Estado de Santa Catarina por meio do Programa Uniedu (Bolsa de Iniciação Científica UNIEDU- Art. 170)

\section{Referências}

Brady, M. C., Jamieson, K., Bugge, C., Hagen, S., McClurg, D., Chalmers, C., \& Langhorne, P. (2016). Caring for continence in stroke care settings: a qualitative study of patients' and staff perspectives on the implementation of a new continence care intervention. Clinical Rehabilitation, 30(5), 481-494.

Bhatia, R., \& Srivastava, M. P. (2020). COVID-19 and stroke: incidental, triggered or causative. Annals of Indian Academy of Neurology, $23(3), 318$.

Brasil. Ministério da Saúde (BR). (2012). Portaria No 665, de 12 de abril de 2012. Dispõe sobre os critérios de habilitação dos estabelecimentos hospitalares como Centro de Atendimento de Urgência aos Pacientes com Acidente Vascular Cerebral (AVC), noâmbito do Sistema Único de Saúde (SUS), institui o respectivo incentivo financeiro e aprova a Linha de Cuidados em AVC. Diário Oficial União. https://bvsms.saude.gov.br/bvs/saudelegis/gm/2012/PRT0665_12_04_2012.html\#: :text=Disp\%C3\%B5e\%20sobre\%20os\%20crit\%C3\%A9rios\%20de,Linha\%2 0de $\% 20$ Cuidados $\% 20 \mathrm{em} \% 20 \mathrm{AVC}$.

Brasil. Ministério da Saúde (BR). (2012). Secretaria de Atenção à Saúde. Departamento de Atenção Básica. Caderno de atenção domiciliar. Departamento de Atenção Básica.

Brasil. Ministério da Saúde (BR). (2013) Secretaria de Atenção à Saúde. Departamento de Ações Programáticas Estratégicas. Diretrizes de atenção à reabilitação da pessoa com acidente vascular cerebral.

Brasil. Ministério da Saúde (BR). (2013). Secretaria de Atenção à Saúde. Departamento de Atenção Básica. Diretrizes para o cuidado das pessoas com doenças crônicas nas redes de atenção à saúde e nas linhas de cuidado prioritárias.

Brasil. Ministério da Saúde (BR). (2020). Secretaria de Atenção Primária à Saúde. Linha de Cuidado do Acidente Vascular Cerebral (AVC) no adulto. 2020. 52 p.

Brasil. Ministério da Saúde (BR). (2020). Secretaria de Atenção Primária à Saúde Departamento de Promoção da Saúde Coordenação-Geral de Prevenção de Doenças Crônicas e Controle do Tabagismo. Manual - como organizar o cuidado de pessoas com doenças crônicas na APS no contexto da pandemia. Brasília DF 2020

Brasil. Ministério da Saúde (BR). (2021). Secretaria de Atenção à Saúde. Sistema de Informações hospitalares. SIHD. 2021. http://sihd.datasus.gov.br/principal/index.php

Brasil. Ministério da Saúde. (2019). Informações de saúde (Tabnet): estatísticas vitais: mortalidade geral. http://tabnet.datasus.gov.br

Buzarelo, F. M., da Silva Rodrigues, T. F. C., Sanches, R. D. C. N., da Silva Rêgo, A., \& Radovanovic, C. A. T. (2018). Aplicação do modelo calgary de avaliação familiar no contexto domiciliar pós acidente vascular encefálico/application of the calgary family assesment model in the home context after encephalic vascular accident. Revista paranaense de enfermagem (REPENF), 1(01).

Chen, L., Xiao, L. D., \& Chamberlain, D. (2020). An integrative review: Challenges and opportunities for stroke survivors and caregivers in hospital to home transition care. Journal of advanced nursing, 76(9), 2253-2265.

Cordeiro, A. M., Oliveira, G. M. D., Rentería, J. M., \& Guimarães, C. A. (2007). Revisão sistemática: uma revisão narrativa. Revista do Colégio Brasileiro de Cirurgiões, 34, 428-431.

Crispim, D., Silva, M. J. P. D., Cedotti, W., Câmara, M., \& Gomes, S. A. (2020). Comunicação difícil e Covid-19-Recomendações práticas para comunicação e acolhimento em diferentes cenários da pandemia.

da Silva Azevedo, P. M. D., Gomes, B. P., Pereira, J. A. T. P., Carvalho, F. M. N., Ferreira, S. P. C., Pires, A. I., \& Macedo, J. (2019). Dependência funcional na alta dos cuidados intensivos: relevância para a enfermagem de reabilitação. Revista de Enfermagem Referência, 4(20), 37-45.

Dusek, B., Pearce, N., Harripaul, A., \& Lloyd, M. (2015). Care transitions: a systematic review of best practices. Journal of nursing care quality, 30(3), 233-239.

Faria, A. R. (2018). Sistema Informatizado de Apoio a Implementação à Linha de Cuidado ao AVC. [Dissertação de Mestrado]. Lages: Mestrado em Ambiente e Saúde, Universidade do Planalto Catarinense.

Faria, A. R., Antunes Baccin, C. R., \& Viapiana Masiero, A. (2019). Estratégias para o enfrentamento do acidente vascular cerebral: reflexões e perspectivas. Atlante Cuadernos de Educación y Desarrollo, (febrero).

Fonseca, L. H. D. O., Rosa, M. L. G., Silva, A. C., Maciel, R. M., Volschan, A., \& Mesquita, E. T. (2013). Análise das barreiras à utilização de trombolíticos em casos de acidente vascular cerebral isquêmico em um hospital privado do Rio de Janeiro, Brasil. Cadernos de Saúde Pública, 29, $2487-2496$.

Fontana, A. P., Lima, F.S. AVC (2020) AVC em meio à pandemia por COVID-19. Guia de cuidados e exercícios fase aguda. Disponível: https://abrafin.org.br/wp-content/uploads/2020/06/avc-pandemia.pdf 
Fox, M. T., Persaud, M., Maimets, I., Brooks, D., O’Brien, K., \& Tregunno, D. (2013). Effectiveness of early discharge planning in acutely ill or injured hospitalized older adults: a systematic review and meta-analysis. BMC geriatrics, 13(1), 1-9.

Gesell, S. B., Klein, K. P., Halladay, J., Bettger, J. P., Freburger, J., Cummings, D. M., ... \& COMPASS Study Investigators. (2017). Methods guiding stakeholder engagement in planning a pragmatic study on changing stroke systems of care. Journal of clinical and translational science, $1(2), 121-128$.

Gibson, J., Coupe, J., \& Watkins, C. (2021). Medication adherence early after stroke: using the Perceptions and Practicalities Framework to explore stroke survivors', informal carers' and nurses' experiences of barriers and solutions. Journal of Research in Nursing, 1744987121993505.

Giles, M. F., \& Rothwell, P. M. (2008). Measuring the prevalence of stroke. Neuroepidemiology, 30(4), 205.

Gracioto, A., Gomes, C. J., Echer, I. C., \& Lorenzi, P. D. C. (2006). Grupo de orientação de cuidados aos familiares de pacientes dependentes. Revista Brasileira de Enfermagem, 59, 105-108.

Kanan, L. A., Masiero, A. V., da Cunha Bellinati, N. V., \& Schonmeier, N. L. A. (2018). Educação e trabalho interprofissional em Saúde: panorama da produção científica brasileira. Atlante Cuadernos de Educación y Desarrollo, noviembre.

Loureiro, A. B. (2020). A Enfermagem de Reabilitação e o planeamento da alta hospitalar (Doctoral dissertation).

Magdon-Ismail, Z., Sicklick, A., Hedeman, R., Bettger, J. P., \& Stein, J. (2016). Selection of postacute stroke rehabilitation facilities: a survey of discharge planners from the northeast cerebrovascular consortium (NECC) region. Medicine, 95(16).

Malta, D. C., Andrade, S. S. C. D. A., Stopa, S. R., Pereira, C. A., Szwarcwald, C. L., Silva, J. B. D., \& Reis, A. A. C. D. (2015). Brazilian lifestyles: National Health Survey results, 2013. Epidemiologia e Serviços de Saúde, 24, 217-226.

Mayor, M. S., Ribeiro, O., \& Paúl, C. (2009). Estudo comparativo: percepção da satisfação de cuidadores de pessoas com demência e cuidadores de pessoas com AVC. Revista Latino-Americana de Enfermagem, 17, 620-624.

Mello, A. I. D. S. (2013). Diretrizes para o plano de alta hospitalar: uma proposta fundamentada no princípio da integralidade.

Monti, L. L., do Nascimento Pinho, J., do Santos, S. C., \& Pinheiro, E. A. (2018). Alta compartilhada pela equipe multiprofissional. Perspectivas Experimentais e Clínicas, Inovações Biomédicas e Educação em Saúde (PECIBES) ISSN-2594-9888, 4(1).

Ntaios, G., Michel, P., Georgiopoulos, G., Guo, Y., Li, W., Xiong, J., ... \& Lip, G. Y. (2020). Characteristics and outcomes in patients with COVID-19 and acute ischemic stroke: the global COVID-19 stroke registry. Stroke, 51(9), e254-e258.

Oliveira, N. R. D. C., Santos, H. F. D. A. C., Garcia, P. T., Pinho, J. R. O., Reis, R. S., Oliveira, A. E. F. D., ... \& França, R. M. (2015). Redes de atenção à saúde: a atenção à saúde organizada em redes.

OPAS - Organização Pan-Americana De Saúde (2020) - Manutenção de serviços essenciais de saúde: orientação operacional para o contexto da COVID-19. Orientação provisória. $1^{\circ}$ de junho de 2020 . Brasilia, D.F.

Pinto, D. M., Jorge, M. S. B., Pinto, A. G. A., Vasconcelos, M. G. F., Cavalcante, C. M., Flores, A. Z. T., \& Andrade, A. S. D. (2011). Projeto terapêutico singular na produção do cuidado integral: uma construção coletiva. Texto \& Contexto-Enfermagem, 20, 493-502.

Rodrigues, F. E. M., \& de Lima, M. M. (2013). Enfrentamento da família durante a hospitalização em unidade de terapia intensiva neonatal/pediátrica. Retirado de: http://www. uniedu. sed. sc. gov. br/wp-content/uploads/2013/10/franciele-euzebio martinsrodrigues. pdf.

Rodrigues, R. A. P., Marques, S., Kusumota, L., Santos, E. B. D., Fhon, J. R. D. S., \& Fabrício-Wehbe, S. C. C. (2013). Transição do cuidado com o idoso após acidente vascular cerebral do hospital para casa. Revista Latino-Americana de Enfermagem, 21, 216-224.

Safanelli, J., Vieira, L. G. D. R., Araujo, T. D., Manchope, L. F. S., Kuhlhoff, M. H. R., Nagel, V., ... \& Cabral, N. L. (2019). The cost of stroke in a public hospital in Brazil: a one-year prospective study. Arquivos de neuro-psiquiatria, 77(6), 404-411.

Santos, L. M. A., de Fátima Morais, R., de Lima, A. K. M., dos Santos Alves, S., \& Barbosa, B. A. O papel da família frente ao paciente com acidente vascular encefálico (ave) após a alta hospitalar: Orientações da equipe de enfermagem. .SBDCV - Sociedade Brasileira de Doenças Cerebrovasculares. 2021

Silva, L. A. T., Soares, L. B., de Souza Reis, C. A., de Gouveia, A. O., de Gouveia, A. O., de Souza, J. R. B., ... \& dos Santos, V. R. C. (2021). Sistematização da Assistência de Enfermagem a um paciente domiciliado com sequela de acidente vascular cerebral. Revista Eletrônica Acervo Saúde, 13 (2), e5513-e5513.

Suksatan, W., \& Posai, V. (2020). An integrative review of discharge planning interventions with Thai stroke patients. Systematic Reviews in Pharmacy, 11(11), 1692-1700.

Vasconcelos, A. M. N., \& Gomes, M. M. F. (2012). Transição demográfica: a experiência brasileira. Epidemiologia e Serviços de Saúde, 21(4), 539-548.

WHO - World Health Organization (2021). Coronavirus disease. situation dashboard presents official daily counts of COVID-19 cases and deaths worldwide. https://covid19.who.int/ 